\title{
A Narrative Review on Dietary Strategies to Provide Nitric Oxide as a Non-Drug Cardiovascular Disease Therapy: Beetroot Formulations-A Smart Nutritional Intervention
}

\author{
Diego dos Santos Baião, Davi Vieira Teixeira da Silva and Vania Margaret Flosi Paschoalin *(D) \\ Chemistry Institute, Federal University of Rio de Janeiro, Avenida Athos da Silveira Ramos 149, \\ Cidade Universitaria, Rio de Janeiro, RJ 21941-909, Brazil; diegobaiao20@ufrj.br (D.d.S.B.); \\ davivieira@ufrj.br (D.V.T.d.S.) \\ * Correspondence: paschv@iq.ufrj.br; Tel.: +55-21-3938-7362; Fax: +55-21-3938-7266
}

check for updates

Citation: dos Santos Baião, D.; Vieira Teixeira da Silva, D.; Margaret Flosi Paschoalin, V. A Narrative Review on Dietary Strategies to Provide Nitric Oxide as a Non-Drug Cardiovascular Disease Therapy: Beetroot Formulations-A Smart Nutritional Intervention. Foods 2021, 10, 859. https://doi.org/10.3390/ foods10040859

Academic Editor: Leslie D. Bourquin

Received: 17 February 2021

Accepted: 13 April 2021

Published: 15 April 2021

Publisher's Note: MDPI stays neutral with regard to jurisdictional claims in published maps and institutional affiliations.

Copyright: (c) 2021 by the authors. Licensee MDPI, Basel, Switzerland. This article is an open access article distributed under the terms and conditions of the Creative Commons Attribution (CC BY) license (https:// creativecommons.org/licenses/by/ $4.0 /)$

\begin{abstract}
Beetroot is a remarkable vegetable, as its rich nitrate and bioactive compound contents ameliorate cardiovascular and metabolic functions by boosting nitric oxide synthesis and regulating gene expressions or modulating proteins and enzyme activities involved in these cellular processes. Dietary nitrate provides a physiological substrate for nitric oxide production, which promotes vasodilatation, increases blood flow and lowers blood pressure. A brief narrative and critical review on dietary nitrate intake effects are addressed herein by considering vegetable sources, dosage, intervention regimen and cardioprotective effects achieved in both healthy and cardiovascularsusceptible individuals. Compared to other nitrate-rich vegetables, beets were proven to be the best choice for non-drug therapy because of their sensorial characteristics and easy formulations that facilitate patient adherence for long periods, allied to bioaccessibility and consequent effectiveness. Beets were shown to be effective in raising nitrate and nitrite in biological fluids at levels capable of promoting sustained improvement in primary and advanced hemodynamic parameters.
\end{abstract}

Keywords: green leaves; beetroot; nitrate-rich diet therapy; nitric oxide; advanced hemodynamic parameters; clinical trials

\section{Introduction}

The vascular endothelium is formed by an endothelial cell monolayer that lines the interior of blood vessels, arteries and veins and cardiac chambers. This protective layer is able to generate an active antithrombotic surface by facilitating the transit of plasma and its cellular constituents throughout the vasculature, regulating the blood flow by maintaining blood vessel tone and hemostasis. The vascular endothelium can play a pivotal role in coagulation cascades and angiogenesis, intracellular signaling regulation and hormone trafficking [1]. Endothelium blood signals release autocrine and paracrine substances in response to diverse stimuli, where angiotensin II, endothelin-1, thromboxane A2 and prostacyclin $\mathrm{H} 2$ participate in vasoconstriction, whereas nitric oxide (NO), bradykinin, and hyperpolarizing factors contribute to vasodilation. Vascular autoregulation influences the structural integrity of vessels and circulation function and hemodynamics [2].

The endothelium-derived NO, the main vasoactive effector, is released in response to physical (sheer stress), hormonal and/or platelet-derived substances stimuli, in addition to vascular relaxation induction, platelet inhibition and leukocyte adhesion, as well as smooth muscle cell proliferation [3].

However, aging, unhealthy lifestyle and eating habits, as well as certain phyisiopathological conditions, including those grouped among the risk factors for cardiovascular disease (CVD), such as obesity, diabetes mellitus and hypercholesteremia, may lead to endothelial dysfunction development [4]. Endothelial dysfunction is considered an event that precedes atherosclerosis, as it causes an imbalance between endothelium-derived 
vasodilators and vasoconstrictor synthesis, resulting in diminished NO production and/or availability [5]. Nutritional and lifestyle interventions capable of restoring NO physiological levels may avoid atherogenesis reducing the risk of cardiovascular diseases, and are considered an effective intervention, mainly in populations presenting poor adherence to pharmacological therapies inherent to chronic diseases. Furthermore, nutritional interventions may also help decrease the costs of public policy healthcare through preventive clinical measures associated with CVD, relieving system burdens. Vegetables are important components of a healthy diet, since they comprise many bioactive compounds termed functional nutrients, providing benefits in the promotion and maintenance of human health $[6,7]$.

Strong evidence suggests that nitrate $\left(\mathrm{NO}_{3}{ }^{-}\right)$, found abundantly in leafy green vegetables, radish and beetroot, after being reduced to nitrite $\left(\mathrm{NO}_{2}{ }^{-}\right)$, is involved in welldocumented cardioprotection, since it provides a physiological substrate for $\mathrm{NO}$ generation through the enterosalivary $\mathrm{NO}_{3}{ }^{-}-\mathrm{NO}_{2}{ }^{-} / \mathrm{NO}$ pathway [8]. However, to exert beneficial human health effects, through full vascular endothelium effects, the long-term dietary intake of $\mathrm{NO}_{3}{ }^{-}$at effective concentration, obtained from food or food-formulations requires popularization. Furthermore, to reach pharmacological $\mathrm{NO}$ concentrations, dietary $\mathrm{NO}_{3}{ }^{-}$intake must be carefully planned by designing high $\mathrm{NO}_{3}{ }^{-}$content formulations from $\mathrm{NO}_{3}{ }^{-}$-rich vegetables, in order to avoid the administration of large serving portions that are mandatory when traditional culinary formulations are used, making it difficult for individuals to adhere to proposed long-term dietary interventions [9].

In this brief narrative and critical review, the role of $\mathrm{NO}$ on endothelial dysfunction and how dietary $\mathrm{NO}_{3}{ }^{-}$can contribute to its generation are described. Furthermore, clinical trials aiming to evaluate health benefits following the intake of dietary $\mathrm{NO}_{3}{ }^{-}$from vegetables will be evaluated and compared, considering the food matrix, ingested content, NO production and consequent improvements in primary and advanced hemodynamic parameters in both healthy individuals and in individuals presenting impaired vascular function.

\section{Nitric Oxide}

$\mathrm{NO}$ is a low molecular weight compound produced in gaseous form, with a short-life and able to diffuse through lipid bilayers, reaching neighboring cells. Its high reactivity character is due to 11 electrons in its valence shell with an unpaired electron, allowing it to rapidly oxidize to $\mathrm{NO}_{2}{ }^{-}$and $\mathrm{NO}_{3}{ }^{-}$[10]. In human physiology, $\mathrm{NO}$ can exert antioxidant functions through its free-radical scavenger ability, thus reducing the rates of reactive oxygen species (ROS) production [11], a harmful superoxide anion $\left(\mathrm{O}_{2}{ }^{\bullet-}\right)$, that generates peroxynitrite $\left(\mathrm{ONOO}^{-}\right)$, able to attack copper and iron-metalloproteins [8]. NO acts as a neurotransmitter in the central and peripheral nervous systems and is able to mediate synapse plasticity in nerve impulse transmission, by favoring the secretion of neurotransmitters or hormones in neuronal junctions [12]. In the cardiovascular system, NO modulates vascular tone by diffusing across endothelial cells, reaching vascular smooth muscle cells and, through soluble guanylate cyclase (sGC), activates the sarcoplasmic calcium $\left(\mathrm{Ca}^{2+}\right)$ pump, decreasing intracellular $\mathrm{Ca}^{2+}$ content and promoting vasodilation as a result of a diminished vascular tone [11,13].

In the human body, $\mathrm{NO}$ is formed from the semi-essential amino acid L-arginine, generating L-citruline as a secondary product. This pathway depends on a group of enzymes, namely nitric oxide synthase (NOS), comprising the neuronal (nNOS or NOS-I) and endothelial (eNOS or NOS-III) isoforms, both constitutive and dependent on the calciumcalmodulin complex, and the inducible isoform (iNOS or NOS-II). NOS requires enzyme cofactors such as nicotinamide adenine dinucleotide phosphate (NADPH), flavin mononucleotide (FMN), flavin adenine dinucleotide (FAD) and tetrahydrobiopterin (BH4) [5]. In addition, the shear stress exerted by blood flow on endothelial cells is crucial for the activation of eNOS under physiological conditions, although other signaling molecules such as 
bradykinin, adenosine, vascular endothelial growth factor (VEGF), and serotonin can also lead to eNOS activation $[10,14]$.

The aforementioned phyisiopathological conditions, i.e., aging associated to an unhealthy lifestyle and the induction of risk factors associated to cardiovascular disease (CVD), such as obesity, diabetes mellitus and hypercholesteremia, which may lead to an imbalance between the synthesis of endothelium-derived vasodilators and vasoconstrictors, may result in diminished NO production and/or availability [5]. This malevolent condition known as endothelial dysfunction precedes atherosclerosis, as the endothelium undergoes structural and functional changes that impair homeostasis and vascular tone maintenance [4]. Oxidative stress and inflammation account for the endothelial dysfunction pathogenesis $[15,16]$. Both physiopathological conditions reduce NO bioavailability through the action of oxidative enzymes such as NADPH oxidase, xanthine oxidase (XO), cyclooxygenases (COX), lipoxygenases (LOX), myeloperoxidases (MPO), cytochrome P450 monooxygenase and peroxidases. In addition to increased oxygen-derived free-radical production and inflammation, eNOS activity and/or expression may decrease due to metabolic impairments [15]. Under these threatening conditions, the endothelium undergoes structural and functional changes, resulting in the exposure of vascular lumen to a prothrombotic and fibrinolytic microenvironment, increasing arterial stiffness and creating favorable conditions for atherosclerosis plaque development [17].

Due to these negative phyisiopathological conditions, the search for healthy diets has been significantly emphasized worldwide [18]. Edible plant matrices contain a complex set of bioactive compounds that may act synergically, making the regular intake of fresh vegetables appealing [19-21]. In order to follow WHO healthy diet recommendations, the worldwide average vegetable consumption, especially in less developed countries, should be of $400 \mathrm{~g} \cdot \mathrm{day}^{-1}$ for $70 \mathrm{~kg}$ individuals (excluding potatoes and other starchy tubers), aiming at reducing the risk of chronic diseases in healthy and unhealthy adults (regardless of age). Among these disorders, CVD is still considered the main cause of morbidity and mortality worldwide [6]. Indeed, meta-analyses studies evaluating health-promoting nutrients have demonstrated that a high vegetable intake is one of the cornerstones of a healthy diet and is recommended to reduce the risk of development coronary heart disease and ischemia, as these food items improve cardiovascular function [22-25]. Indeed, a recent systematic review and meta-analysis calculated the summary relative risk (RR) of incidence or mortality considering a $200 \mathrm{~g} \cdot \mathrm{day}^{-1}$ intake of a combination of vegetables. The RR for coronary heart disease was of 0.84 (95\% CI: $\left.0.79-0.90, \mathrm{I}^{2}=61 \%, n=15\right)$, the same as for stroke, $0.84\left(95 \%\right.$ CI: 0.76-0.92, $\left.\mathrm{I}^{2}=73 \%, n=10\right)$, increasing to 0.92 (95\% CI: $0.90-0.95$, $\mathrm{I}^{2}=31 \%, n=13$ ) for cardiovascular disease. Similar relative cardiovascular malfunction risks were observed for fruit or vegetable intakes, separately [23].

\section{Dietary $\mathrm{NO}_{3}{ }^{-}$and Endothelial Dysfunction Therapy}

Until a decade ago, $\mathrm{NO}_{3}{ }^{-}$was considered an unfavorable dietary-derived toxic compound, as it was wrongly associated with the development of some malignancies, such as gastric cancer. Strict standards regarding the levels of this anion were regulated in food [26]. The World Health Organization (WHO) defined an acceptable daily intake (ADI) of $3.7 \mathrm{mg}$ of $\mathrm{NO}_{3}{ }^{-} \cdot \mathrm{kg}^{-1}$ body weight, the same content adopted by the European Food Safety Authority. For a normal adult weighing $70 \mathrm{~kg}$, this content is equivalent to $\sim 260 \mathrm{mg}$ of $\mathrm{NO}_{3}{ }^{-} \cdot$ day $^{-1}$. However, vegetarian diets commonly contain $>300 \mathrm{mg}$ of $\mathrm{NO}_{3}{ }^{-} \cdot$ day $^{-1}$ for $70 \mathrm{~kg}$ adults, higher than the ADI [27].

Recently, researchers have become interested in the biological $\mathrm{NO}_{3}{ }^{-}$role. Findings regarding the improvement of cardiovascular function have raised a biologically plausible and widely recognized hypothesis that the $\mathrm{NO}_{3}{ }^{-}$present in vegetables may serve as a physiological substrate for NO generation which, in turn, promotes vasodilation and, consequently, improves cardiovascular function $[8,13]$.

$\mathrm{NO}_{3}{ }^{-}$is a nitric acid salt, while $\mathrm{NO}_{2}{ }^{-}$is a nitrous acid salt compound, formed by a single nitrogen bonded to three or two oxygen atoms, respectively. Both compounds can 
be obtained from endogenous and/or exogenous sources. The endogenous formation of $\mathrm{NO}_{3}{ }^{-}$and $\mathrm{NO}_{2}{ }^{-}$occurs through the $\mathrm{NO}$ metabolism via the L-arginine/NO pathway, as mentioned previously. On the other hand, the main potential exogenous source for the acquisition of $\mathrm{NO}_{3}{ }^{-}$and $\mathrm{NO}_{2}{ }^{-}$is through the dietary route. Through this pathway, $\mathrm{NO}$ is then generated by a non-enzymatic pathway from $\mathrm{NO}_{2}{ }^{-}$. Dietary $\mathrm{NO}_{3}{ }^{-}$is reduced to $\mathrm{NO}_{2}{ }^{-}$in the oral cavity by bacteria that produce the $\mathrm{NO}_{3}{ }^{-}$-reductase enzyme [9]. The metabolic activities of commensal bacteria species, such as Granulicatella, Actinomyces, Veillonella, Prevotella, Neisseria, Haemophilus, and Rothia genera that inhabit the oral cavity have a significant influence on $\mathrm{NO}_{3}{ }^{-}$to $\mathrm{NO}$ pathway. Previous studies have shown that individuals with a higher abundance of $\mathrm{NO}_{3}{ }^{-}$-reducing bacteria are able to generate more salivary $\mathrm{NO}_{2}{ }^{-}$and, consequently, $\mathrm{NO}$, at a faster rate following dietary $\mathrm{NO}_{3}{ }^{-}$ ingestion [28]. However, enzymatic activity in the mouth and, consequently, the conversion of $\mathrm{NO}_{3}{ }^{-}$to $\mathrm{NO}_{2}{ }^{-}$may be disrupted by antibiotic use or mouthwash rinsing, since both substances inactivate bacteria cells [10]. Subsequently, $\mathrm{NO}_{2}{ }^{-}$reaches the stomach and, in this acidic environment, is protonated, forming nitrous acid $\left(\mathrm{HNO}_{2}\right)$, which decomposes non-enzymatically to $\mathrm{NO}$ and other bioactive nitrogen oxides such as nitrogen dioxide $\left(\mathrm{NO}_{2}\right)$, dinitrogen trioxide $\left(\mathrm{N}_{2} \mathrm{O}_{3}\right)$ and the nitrosonium ion $\left(\mathrm{NO}^{+}\right)[9,13]$. The remaining $\mathrm{NO}_{3}{ }^{-}$and $\mathrm{NO}_{2}{ }^{-}$in the jejunum are rapidly absorbed into the bloodstream or tissues, where their accumulation occurs in tandem with molecules endogenously synthesized by the L-arginine/NO pathway. Most $\mathrm{NO}_{3}{ }^{-}$is excreted in urine, whereas a small portion is extracted by the salivary glands, concentrating this compound in the saliva, continuing the entero-salivary cycle $[8,9]$. A small part of plasma $\mathrm{NO}_{3}{ }^{-}$and $\mathrm{NO}_{2}{ }^{-}$concentrations may suffer the action of xanthine oxidoreductase (XOR), which displays similar activity to $\mathrm{NO}_{3}{ }^{-}$reductase. $\mathrm{NO}_{2}{ }^{-}$can also be reduced to bioactive $\mathrm{NO}$ by deoxyhemoglobin (deoxyHb) and deoxymyoglobin (deoxyMb), especially when $\mathrm{O}_{2}$ levels are low. Other enzymes and compounds exhibiting redox potential, such as aldehyde oxidase (AO), aldehyde dehydrogenase (ALDH), carbonic anhydrase (CA), vitamin C (Vit C.) and polyphenols, display the ability to synthesize $\mathrm{NO}$ from $\mathrm{NO}_{2}{ }^{-}$reduction [8].

Several studies report beneficial effects of dietary $\mathrm{NO}_{3}{ }^{-}$sources as a new physiological, therapeutic and nutritional approach to attain the intended cardioprotective effects by NO production stimulation $[8,29,30]$. However, dosage, supplementation regimen and individual health status must be considered to obtain the maximum cardioprotective effect following $\mathrm{NO}_{3}{ }^{-}$intake. Furthermore, environmental factors such as temperature, exposure to sunlight, atmospheric humidity, water content and irradiation, as well as agricultural factors like plant genotype, fertilization, herbicide use, amount of available nitrogen, type of crop, soil conditions, nutrient availability and transport and, finally, storage conditions also influence $\mathrm{NO}_{3}{ }^{-}$contents in plants, and, consequently dietary $\mathrm{NO}_{3}{ }^{-}$supplementation [31].

\section{Dietary $\mathrm{NO}_{3}{ }^{-}$Vegetable Sources}

Vegetables are the main source of dietary $\mathrm{NO}_{3}{ }^{-}$, corresponding to $85 \%$ of the daily intake, although $\mathrm{NO}_{3}{ }^{-}$content can vary widely within members of distinct botanical families [32]. The $\mathrm{NO}_{3}{ }^{-}$contents in plant organs can be classified from highest to lowest, as petiole $>$ leaf $>$ stem $>$ root $>$ tuber $>$ bulb $>$ fruit $>$ seed [33]. Table 1 presents a list of vegetables commonly included in Western diets considered $\mathrm{NO}_{3}{ }^{-}$sources, classified according to $\mathrm{NO}_{3}{ }^{-}$contents, from the highest to the lowest. 
Table 1. Dietary $\mathrm{NO}_{3}{ }^{-}$sources classified from the highest to the lowest according to mean [and range] $\mathrm{NO}_{3}{ }^{-}$content.

\begin{tabular}{|c|c|c|}
\hline & Vegetable & $\mathrm{NO}_{3}-$ Content $/ \mathrm{mg} \cdot \mathrm{kg}^{-1}$ \\
\hline \multirow{17}{*}{$\begin{array}{l}\text { High } \mathrm{NO}_{3}{ }^{-} \text {content } \\
\left(>1000 \mathrm{mg} \cdot \mathrm{kg}^{-1}\right)\end{array}$} & Rocket or arugula (Eruca vesicaria subsp. sativa) & 2848 [2597-3100] \\
\hline & Green spinach (Spinacia oleracea) & 2500 [2013-2797] \\
\hline & Coriander (Coriandrum sativum) & 2445 \\
\hline & Basil (Ocimum basilicum) & $2292[507-4695]$ \\
\hline & Celery (Apium graveolens) & $2200[900-3500]$ \\
\hline & Parsley (Petroselinum crispum) & $2134[1700-2101]$ \\
\hline & Radish (Raphanus raphanistrum subsp. sativus) & $2064[1878-2250]$ \\
\hline & Butter leaf lettuce (Lactuca sativa variety capitata) & 2000 \\
\hline & Bok choy (Brassica rapa subsp. chinensis) & 1933 \\
\hline & Lettuce (Lactuca sativa) & $1893[970-2782]$ \\
\hline & Beet greens (Beta vulgaris subsp. vulgaris) & $1852[1060-2600]$ \\
\hline & Kohlrabi (Brassica oleracea) & 1769 \\
\hline & Swiss chard (Beta vulgaris subsp. maritima) & 1512 [1024-2000] \\
\hline & Leaf chicory (Cichorium intybus) & 1452 \\
\hline & Beetroot (Beta vulgaris subsp. vulgaris) & $1300[644-1950]$ \\
\hline & Black radish (Raphanus raphanistrum subsp. sativus) & $1271[667-1878]$ \\
\hline & Mustard greens (Brassica juncea) & 1160 \\
\hline \multirow{22}{*}{$\begin{array}{l}\text { Medium } \mathrm{NO}_{3}{ }^{-} \text {content } \\
\left(100 \text { to } 1000 \mathrm{mg} \cdot \mathrm{kg}^{-1}\right)\end{array}$} & Curly kale (Brassica oleracea Acephala Group) & 987 [792-1181] \\
\hline & Broccoli raab (Brassica rapa) & 905 \\
\hline & Pumpkin (Cucurbita pepo) & $692[445-939]$ \\
\hline & Turnip (Brassica rapa subsp. rapa) & $684[307-1062]$ \\
\hline & Endive (Cichorium endivia) & 663 \\
\hline & Cabbage (Brassica oleracea var. capitata) & 503 [85-920] \\
\hline & Green beans (Phaseolus vulgaris) & $496[449-585]$ \\
\hline & Green onion (Allium fistulosum) & 485 [99-870] \\
\hline & Courgette (Cucurbita pepo) & 416 \\
\hline & Fennel (Foeniculum vulgare) & 363 \\
\hline & Asparagus (Asparagus officinalis) & 355 [145-479] \\
\hline & Cauliflower (Brassica oleracea var. botrytis) & $331[104-559]$ \\
\hline & Savoy cabbage (Brassica oleracea Savoy Cabbage Group) & 324 \\
\hline & Aubergine (Solanum melongena) & 314 \\
\hline & Broccoli (Brassica oleracea var. italica) & 300 [145-477] \\
\hline & Carrot (Daucus carota subsp. sativus) & $300[121-480]$ \\
\hline & Cucumber (Cucumis sativus) & $240[124-384]$ \\
\hline & Potato (Solanum tuberosum) & $220[81-713]$ \\
\hline & Garlic (Allium sativum) & 183 [34-455] \\
\hline & Artichokes (Cynara scolymus) & 174 \\
\hline & Sweet pepper (Capsicum annuum) & 117 [93-140] \\
\hline & Green pepper (Capsicum annuum) & $111[76-159]$ \\
\hline \multirow{2}{*}{ Low $\mathrm{NO}_{3}{ }^{-}$content $\left(<100 \mathrm{mg} \cdot \mathrm{kg}^{-1}\right)$} & Onion (Allium cepa) & 87 [23-235] \\
\hline & Tomato (Solanum lycopersicum) & $69[27-170]$ \\
\hline
\end{tabular}

$\mathrm{NO}_{3}{ }^{-}$vegetables content were compiled from Lidder and Webb [8]; Hord et al. [33]; Santamaria et al. [34]; EFSA [35] and Tamme et al. [36].

The $\mathrm{NO}_{3}{ }^{-}$-rich vegetables within the Amaranthaceae family comprise beetroot $\left(1300 \mathrm{mg} \cdot \mathrm{kg}^{-1}\right)$, beet greens $\left(1852 \mathrm{mg} \cdot \mathrm{kg}^{-1}\right)$, Swiss chard $\left(1690 \mathrm{mg} \cdot \mathrm{kg}^{-1}\right)$, and green spinach $\left(\approx 2500 \mathrm{mg} \cdot \mathrm{kg}^{-1}\right)$, while a Lamiaceae family representative consists of basil (2292 $\left.\mathrm{mg} \cdot \mathrm{kg}^{-1}\right)$. Concerning the Brassicaceae family, the most representative members are bok choy $\left(1933 \mathrm{mg} \cdot \mathrm{kg}^{-1}\right)$, black radish $\left(1271 \mathrm{mg} \cdot \mathrm{kg}^{-1}\right)$, turnip $\left(1018 \mathrm{mg} \cdot \mathrm{kg}^{-1}\right)$, mustard greens $\left(1160 \mathrm{mg} \cdot \mathrm{kg}^{-1}\right)$, rocket or arugula $\left(4677 \mathrm{mg} \cdot \mathrm{kg}^{-1}\right)$, kohlrabi $\left(1769 \mathrm{mg} \cdot \mathrm{kg}^{-1}\right)$, and radish $\left(\approx 2000 \mathrm{mg} \cdot \mathrm{kg}^{-1}\right)$. Apiaceae family members include coriander $\left(2445 \mathrm{mg} \cdot \mathrm{kg}^{-1}\right)$, celery $\left(1100 \mathrm{mg} \cdot \mathrm{kg}^{-1}\right)$ and parsley $\left(2134 \mathrm{mg} \cdot \mathrm{kg}^{-1}\right)$, whereas Asteraceae family members include lettuce $\left(\approx 1800 \mathrm{mg} \cdot \mathrm{kg}^{-1}\right)$, leaf chicory $\left(1452 \mathrm{mg} \cdot \mathrm{kg}^{-1}\right)$, and butter leaf lettuce $\left(2000 \mathrm{mg} \cdot \mathrm{kg}^{-1}\right)$. All these vegetables are included in the high $\mathrm{NO}_{3}{ }^{-}$-containing vegetable category of $>1000 \mathrm{mg} \cdot \mathrm{kg}^{-1}$. Vegetables such as cabbage $\left(513 \mathrm{mg} \cdot \mathrm{kg}^{-1}\right)$, curly kale $\left(987 \mathrm{mg} \cdot \mathrm{kg}^{-1}\right)$, broccoli $\left(\approx 300 \mathrm{mg} \cdot \mathrm{kg}^{-1}\right)$, broccoli raab $\left(905 \mathrm{mg} \cdot \mathrm{kg}^{-1}\right)$, cauliflower $\left(202 \mathrm{mg} \cdot \mathrm{kg}^{-1}\right)$ and Savoy 
cabbage (324 mg $\left.\cdot \mathrm{kg}^{-1}\right)$, which belong to the Brassicaceae family; carrot $\left(\approx 300 \mathrm{mg} \cdot \mathrm{kg}^{-1}\right)$ and fennel $\left(363 \mathrm{mg} \cdot \mathrm{kg}^{-1}\right)$, both members of the Apiaceae family; artichokes $\left(174 \mathrm{mg} \cdot \mathrm{kg}^{-1}\right)$, asparagus chicory $\left(355 \mathrm{mg} \cdot \mathrm{kg}^{-1}\right)$, and endive $\left(663 \mathrm{mg} \cdot \mathrm{kg}^{-1}\right)$, belonging to the Asteraceae family, garlic $\left(183 \mathrm{mg} \cdot \mathrm{kg}^{-1}\right)$ and green onion $\left(\approx 450 \mathrm{mg} \cdot \mathrm{kg}^{-1}\right)$ from the Liliaceae family; aubergine $\left(314 \mathrm{mg} \cdot \mathrm{kg}^{-1}\right)$, capsicum $\left(108 \mathrm{mg} \cdot \mathrm{kg}^{-1}\right)$ and potato $\left(220 \mathrm{mg} \cdot \mathrm{kg}^{-1}\right)$, belonging Solanaceae family; courgette $\left(416 \mathrm{mg} \cdot \mathrm{kg}^{-1}\right)$ and cucumber $\left(240 \mathrm{mg} \cdot \mathrm{kg}^{-1}\right)$, pumpkin (894 mg $\cdot \mathrm{kg}^{-1}$ ), from the Cucurbitaceae family member all contain intermediate $\mathrm{NO}_{3}{ }^{-}$concentrations ranging from 100 to $1000 \mathrm{mg} \cdot \mathrm{kg}^{-1}$ [33-36].

Among the vegetables considered the richest dietary $\mathrm{NO}_{3}{ }^{-}$sources, as listed in Table 1, beetroot, rocket and spinach have been the most tested concerning dietary interventions, and all resulted in effective improvements in cardiovascular performance estimated through blood pressure reduction and vascular function amelioration (Figure 1).

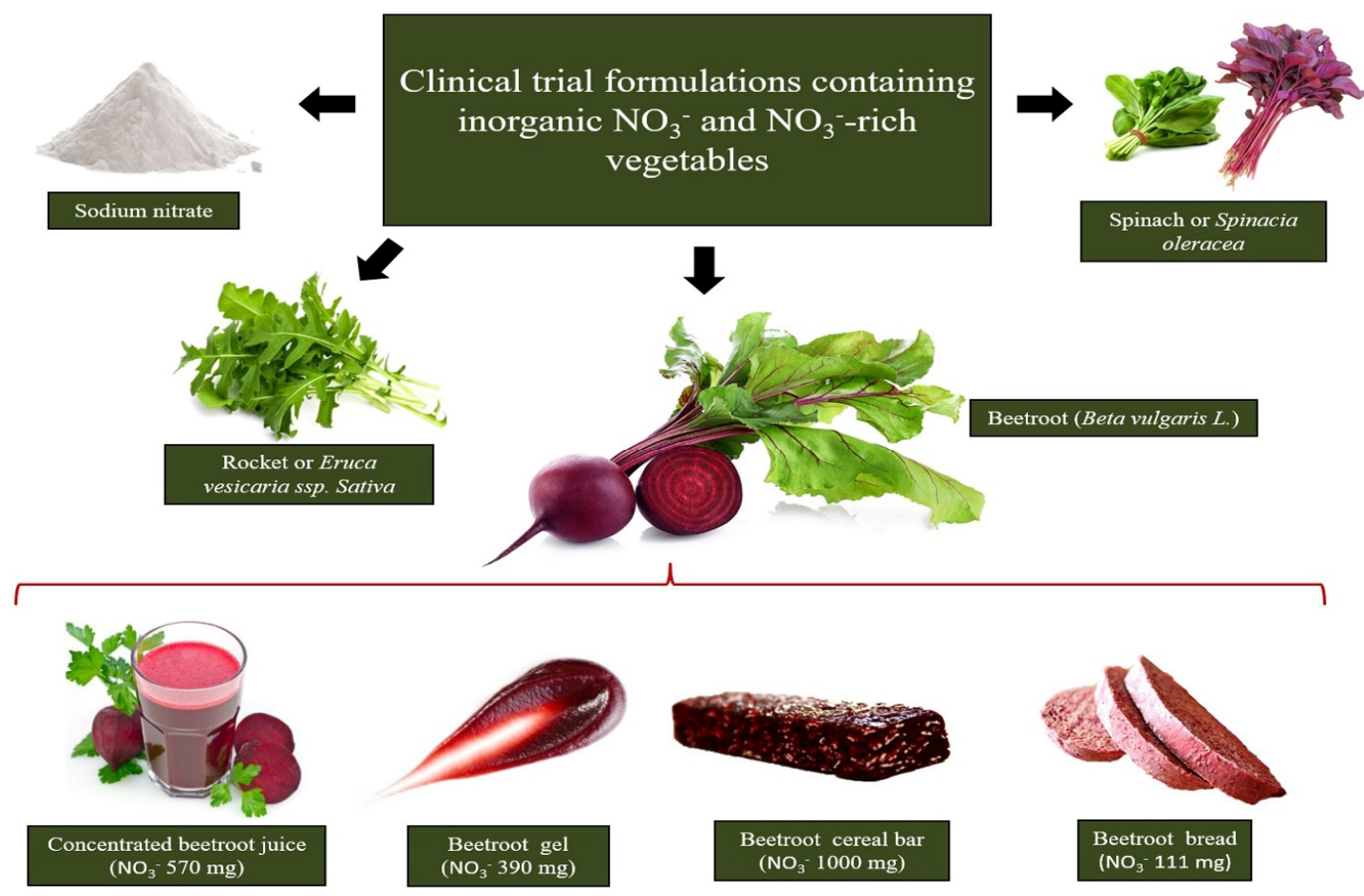

Figure 1. The richest sources of dietary $\mathrm{NO}_{3}{ }^{-}$tested in clinical interventions are beetroot, rocket and spinach. Beetroot formulation choice to supplement dietary $\mathrm{NO}_{3}{ }^{-}$relies on the design of beetroot-derived formulations containing pharmacological $\mathrm{NO}_{3}{ }^{-}$doses in a small serving portion.

$\mathrm{NO}_{3}{ }^{-}$vascular-effects depend on digestibility and bioavailability (bioacessibility), and better performances are obtained when $\mathrm{NO}_{3}{ }^{-}$intake originates from food matrices compared to $\mathrm{NaNO}_{3}{ }^{-}$salt administration [37]. The beneficial effects of different $\mathrm{NO}_{3}{ }^{-}$-rich vegetables and $\mathrm{NO}_{3}{ }^{-}$doses in $\mathrm{NO}$ stimulation production and biochemical, hemodynamic, and vascular parameters in healthy or cardiovascular-compromised patients are summarized in Table 2. It is important to note that, to the best of our knowledge, $\mathrm{NO}_{3}{ }^{-}$ supplementation from green leaves has only been performed in healthy individuals, and it is unknown whether their effects can be extended to individuals presenting cardiovascular risk factors. In addition, although the cardiovascular protective effects of $\mathrm{NO}_{3}{ }^{-}$-enriched vegetables have been clearly demonstrated in clinical trials with healthy individuals, the large volume of juice vegetables used to achieve effective dietary $\mathrm{NO}_{3}{ }^{-}$concentrations can be a limiting factor in ensuring adherence to long-term nutritional interventions. However, this $\mathrm{NO}_{3}{ }^{-}$limitation does not impact supplementation by beetroot juice. Beet juice and other beetroot formulations can be ingested in comfortable serving portions to achieve threshold $\mathrm{NO}_{3}{ }^{-}$concentrations in order to promote beneficial cardiovascular function effects. 


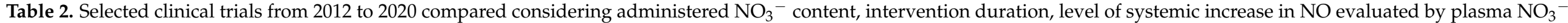
and $\mathrm{NO}_{2}{ }^{-}$levels and improvements in primary and advanced hemodynamic parameters in healthy individuals and in patients presenting impaired vascular function.

\begin{tabular}{|c|c|c|c|c|c|c|}
\hline $\begin{array}{l}\mathrm{NO}_{3}^{-} \text {Vegetable } \\
\text { Intervention }\end{array}$ & $\begin{array}{l}\mathrm{NO}_{3}-\text { Content/Serving } \\
\text { Portion Administered }\end{array}$ & Subjects & $\begin{array}{c}\text { Duration of } \\
\text { Administration }\end{array}$ & Trial Features & Effects & Study \\
\hline $\begin{array}{l}\text { White beetroot bread } \\
\text { (Beta vulgaris L) } \\
\text { Red beetroot bread } \\
\text { (Beta vulgaris L) }\end{array}$ & $\begin{array}{c}99 \mathrm{mg} \cdot 200 \mathrm{~g}^{-1} \\
112 \mathrm{mg} \cdot 200 \mathrm{~g}^{-1}\end{array}$ & 14 healthy individuals & single intake & $\begin{array}{l}\text { Randomized } \\
\text { Placebo-controlled } \\
\text { Single-blind } \\
\text { Crossover }\end{array}$ & $\begin{array}{c}\uparrow \mathrm{NO} \text { synthesis after } 1 \mathrm{~h} \text { of } \\
\text { ingestion (through urinary NO } \mathrm{NO}_{\mathrm{x}} \\
\downarrow 24 \mathrm{~h} \text { ambulatory SBP and DBP }\end{array}$ & Hobbs et al. [38] \\
\hline $\begin{array}{l}\text { Beetroot bread (Beta } \\
\text { vulgaris L) }\end{array}$ & $68 \mathrm{mg} \cdot 200 \mathrm{~g}^{-1}$ & 23 healthy individuals & single intake & $\begin{array}{l}\text { Randomized } \\
\text { Placebo-controlled } \\
\text { Open-label } \\
\text { Crossover }\end{array}$ & $\begin{array}{c}\uparrow \mathrm{NO} \text { synthesis after } 1 \mathrm{~h} \text { of } \\
\text { ingestion (through plasma and } \\
\text { urinary } \mathrm{NO}_{3}-\text { and } \mathrm{NO}_{2}^{-} \text {) } \\
\downarrow \text { iAUC }(0-6 \mathrm{~h} \text { after beet bread } \\
\text { ingestion) for DBP } \\
\uparrow \text { iAUC (0-6 } \mathrm{h} \text { after beet bread } \\
\text { ingestion) for } \\
\text { endothelium-independent } \\
\text { microvascular vasodilation }\end{array}$ & Hobbs et al. [39] \\
\hline \multirow{4}{*}{$\begin{array}{l}\text { Beetroot juice } \\
\text { (Beta vulgaris } \mathrm{L} \text { ) }\end{array}$} & $403 \mathrm{mg} \cdot 70 \mathrm{~mL}^{-1}$ & $\begin{array}{l}24 \text { overweight older } \\
\text { individuals }\end{array}$ & 3 weeks & $\begin{array}{c}\text { Randomized } \\
\text { Placebo-controlled }\end{array}$ & $\downarrow$ daily resting DBP at home & Jajja et al. [40] \\
\hline & $400 \mathrm{mg} \cdot 250 \mathrm{~mL}^{-1}$ & $\begin{array}{l}68 \text { hypertensive } \\
\text { individuals }\end{array}$ & 4 weeks & $\begin{array}{l}\text { Randomized } \\
\text { Placebo-controlled } \\
\text { Double-blind } \\
\text { Crossover }\end{array}$ & $\begin{array}{l}\uparrow \mathrm{NO} \text { synthesis (by plasma and } \\
\text { salivary } \mathrm{NO}_{3}^{-}, \mathrm{NO}_{2}^{-} \text {and } \\
\text { plasma cGMP) } \\
\downarrow \text { home, clinic and } 24 \mathrm{~h} \\
\text { ambulatorial SBP and DBP, and } \\
\text { arterial stiffness (through } \\
\text { reduction of PWV and AIx) } \\
\uparrow \text { endothelial function (through } \\
\text { increased brachial artery } \\
\text { diameter and time to peak } \\
\text { dilatation after FMD) }\end{array}$ & Kapil et al. [41] \\
\hline & $100 \mathrm{mg} \cdot 100 \mathrm{~mL}^{-1}$ & 40 healthy individuals & single intake & $\begin{array}{l}\text { Randomized } \\
\text { Placebo-controlled } \\
\text { Double-blind } \\
\text { Crossover }\end{array}$ & $\begin{array}{c}\uparrow \mathrm{NO} \text { synthesis (by urinary } \mathrm{NO}_{3}^{-} \\
\text {and } \mathrm{NO}_{2}^{-} \text {) } \\
\text { No significant relationships } \\
\text { between urinary } \mathrm{NO}_{3}^{-} \text {and } \\
\mathrm{NO}_{2}{ }^{-} \text {concentration and body } \\
\text { mass after intervention were } \\
\text { observed }\end{array}$ & Baião et al. [42] \\
\hline & $800 \mathrm{mg} \cdot 200 \mathrm{~mL}^{-1}$ & $\begin{array}{l}14 \text { non-hypertensive } \\
\text { obese individuals }\end{array}$ & single intake & $\begin{array}{c}\text { Randomized } \\
\text { Placebo-controlled } \\
\text { Crossover }\end{array}$ & $\begin{array}{c}\uparrow \text { NO synthesis (through plasma } \\
\text { NO } \\
\downarrow \\
\downarrow \text { ambulatory SBP following 1-6 } \\
\text { h of moderate-intensity aerobic } \\
\text { exercise }\end{array}$ & Bezerra et al. [43] \\
\hline
\end{tabular}


Table 2. Cont.

\begin{tabular}{|c|c|c|c|c|c|c|}
\hline $\begin{array}{l}\mathrm{NO}_{3}-\text { Vegetable } \\
\text { Intervention }\end{array}$ & $\begin{array}{l}\mathrm{NO}_{3}-\text { Content/Serving } \\
\text { Portion Administered }\end{array}$ & Subjects & $\begin{array}{c}\text { Duration of } \\
\text { Administration }\end{array}$ & Trial Features & Effects & Study \\
\hline $\begin{array}{c}\text { Beetroot gel } \\
\text { (Beta vulgaris L) }\end{array}$ & $390 \mathrm{mg} \cdot 100 \mathrm{~g}^{-1}$ & 5 healthy individuals & single intake & - & $\begin{array}{l}\uparrow \mathrm{NO} \text { synthesis (through plasma } \\
\mathrm{NO}_{2}^{-} \text {) } \\
\downarrow \text { ambulatory SBP, DBP and HR }\end{array}$ & Silva et al. [44] \\
\hline $\begin{array}{l}\text { Beetroot cereal bar } \\
\text { (Beta vulgaris L) }\end{array}$ & $589 \mathrm{mg} \cdot 60 \mathrm{~g}^{-1}$ & $\begin{array}{l}\text { women with } 2 \text { risk factors } \\
\text { for CVD }\end{array}$ & 3 weeks & $\begin{array}{l}\text { Randomized } \\
\text { Placebo-controlled } \\
\text { Double-blind } \\
\text { Crossover }\end{array}$ & $\begin{array}{c}\uparrow \mathrm{NO} \text { synthesis (through plasma } \\
\mathrm{NO}_{3}{ }^{-} \text {and } \mathrm{NO}_{2}^{-} \text {) } \\
\downarrow \text { clinical DBP and SBP } \\
\downarrow \text { arterial stiffness (through } \\
\text { reductions in AP, AIx, aoSP, ao PP, } \\
\text { arterial age and PWV) } \\
\uparrow \text { endothelial function (through } \\
\text { increased CVC peaks and AUC) }\end{array}$ & Baião et al. [45] \\
\hline \multirow{2}{*}{$\begin{array}{c}\text { Spinach } \\
\text { (Spinacia oleracea) }\end{array}$} & $220 \mathrm{mg} \cdot 250 \mathrm{~g}^{-1}$ & 26 healthy individuals & single intake & $\begin{array}{l}\text { Randomized } \\
\text { Placebo-controlled } \\
\text { Crossover }\end{array}$ & $\begin{array}{l}\uparrow \mathrm{NO} \text { synthesis (through salivary } \\
\mathrm{NO}_{3}{ }^{-} \text {and } \mathrm{NO}_{2}^{-} \text {) } \\
\uparrow \text { large artery elasticity index } \\
\downarrow \text { pulse pressure, SBP, estimated } \\
\text { cardiac ejection time, estimated } \\
\text { cardiac output, estimated stroke } \\
\text { volume and total vascular } \\
\text { impedance }\end{array}$ & Liu et al. [46] \\
\hline & $800 \mathrm{mg} \cdot 365 \mathrm{~g}^{-1}$ & 18 healthy individuals & single intake & $\begin{array}{l}\text { Semi randomized } \\
\text { Crossover }\end{array}$ & $\begin{array}{c}\uparrow \mathrm{NO} \text { synthesis (through plasma } \\
\mathrm{NO}_{3}^{-} \text {and } \mathrm{NO}_{2}^{-} \text {) } \\
\downarrow \text { ambulatory DBP and SBP }\end{array}$ & Jonvik et al. [48] \\
\hline $\begin{array}{c}\text { Red spinach } \\
\text { (Amaranthus dubius) }\end{array}$ & $1000 \mathrm{mg} \cdot 90 \mathrm{mg}^{-1}$ & 15 healthy individuals & single intake & $\begin{array}{l}\text { Placebo-controlled } \\
\text { Double-blind } \\
\text { Crossover }\end{array}$ & $\begin{array}{l}\uparrow \mathrm{NO} \text { synthesis (through plasma } \\
\mathrm{NO}_{2}{ }^{-} \text {and } \mathrm{NO}_{\mathrm{x}} \text { ) } \\
\uparrow \text { endothelial function (through } \\
\text { increased reactive hyperemia and } \\
\text { calf blood flow) }\end{array}$ & Haun et al. [49] \\
\hline $\begin{array}{c}\text { Rocket (Euruca } \\
\text { vesicaria ssp. Sativa) }\end{array}$ & $800 \mathrm{mg} \cdot 196 \mathrm{~g}^{-1}$ & 18 healthy individuals & single intake & $\begin{array}{l}\text { Semi randomized } \\
\text { Crossover }\end{array}$ & $\begin{array}{c}\uparrow \mathrm{NO} \text { synthesis (through plasma } \\
\mathrm{NO}_{3}^{-} \text {and } \mathrm{NO}_{2}^{-} \text {) } \\
\downarrow \text { DBP and } \mathrm{SBP}^{-}\end{array}$ & Jonvik et al. [48] \\
\hline
\end{tabular}

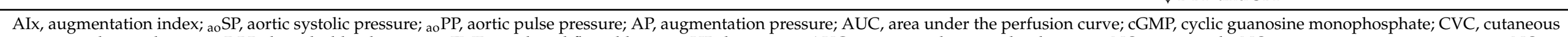

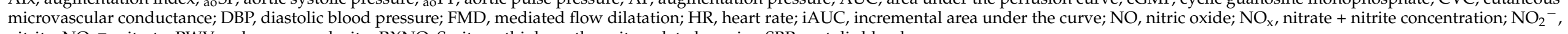
nitrite; $\mathrm{NO}_{3}{ }^{-}$, nitrate; $\mathrm{PWV}$, pulse wave velocity; $\mathrm{RXNO}$, S-nitrosothiols + other nitrosylated species; SBP, systolic blood pressure. 
A large volume of spinach comprising a serving portion of $250 \mathrm{~g}$ leaves containing $220 \mathrm{mg}$ of $\mathrm{NO}_{3}{ }^{-}$were administrated to twenty-six healthy individuals, resulting in an increase in $\mathrm{NO}$ synthesis evidenced by an eight-fold increase in salivary $\mathrm{NO}_{2}{ }^{-}$and a sevenfold increase in salivary $\mathrm{NO}_{3}{ }^{-}$at 120 min post-meal. Large artery elasticity indices were increased alongside lower pulse pressure and reduced systolic blood pressure (SBP) [46].

An amount of $800 \mathrm{mg} \mathrm{NO}_{3}{ }^{-}$intake was supplied through four different vegetable drinks, namely beetroot juice (116 g), rocket salad (196 g), spinach (365 g) or $\mathrm{NaNO}_{3}{ }^{-}(1.1 \mathrm{~g})$ prepared in water, which triggered an increase in $\mathrm{NO}_{3}{ }^{-}$and $\mathrm{NO}_{2}{ }^{-}$plasma concentrations. SBP declined after $150 \mathrm{~min}$ of beetroot juice ingestion (from $118 \pm 2$ to $113 \pm 2 \mathrm{~mm} \mathrm{Hg}$ ) and a rocket salad beverage (from $122 \pm 3$ to $116 \pm 2 \mathrm{~mm} \mathrm{Hg}$ ), which was sustained for at least $300 \mathrm{~min}$ after ingestion of the spinach beverage (from $118 \pm 2$ to $111 \pm 3 \mathrm{~mm} \mathrm{Hg}$ ). Diastolic blood pressure (DBP) also declined after 150 min ingestion of all beverages and was sustained at lower levels for $300 \mathrm{~min}$ after rocket salad or spinach ingestion [48].

All $\mathrm{NO}_{3}{ }^{-}$rich-vegetable drinks were more efficient than $\mathrm{NaNO}_{3}{ }^{-}$in reducing both SBP and DBP, and beetroot was the most effective considering the food weight $/ \mathrm{NO}_{3}{ }^{-}$ content ratio [48]. However, to the best of our knowledge, the lowest effective volume of beetroot able to promote beneficial vascular effects was $70 \mathrm{~mL}$ of beetroot juice containing $6.45 \mathrm{mmol} \mathrm{NO}_{3}{ }^{-}$(403 $\mathrm{mg}$ ), which was administered to 24 older and overweight volunteers for three weeks. This supplementation regimen and the offered dose promoted 2.3-fold and 7.3-fold increases in urinary and salivary $\mathrm{NO}_{3}{ }^{-}$, respectively, and resulted in a $7.3 \mathrm{~mm}$ $\mathrm{Hg}$ decrease in SBP [50].

Beetroot consumption is noteworthy as a convenient and attractive alternative to obtain cardioprotective $\mathrm{NO}_{3}{ }^{-}$effects in both healthy individuals and those presenting risk factors for CVD diseases, due to the distinct but smart formulations (traditional or novel) that can be prepared to fulfill effective pharmacological dietary $\mathrm{NO}_{3}{ }^{-}$concentrations. An attractive and compact $\mathrm{NO}_{3}{ }^{-}$-enriched-beetroot gel has been formulated in an attempt to provide an enriched $\mathrm{NO}_{3}{ }^{-}$food product able to promote the claimed cardioprotective effects while still being easy to administer and facilitate adherence to nutritional therapy [44]. Acute supplementation with $100 \mathrm{~g}$ of beetroot gel containing $390 \mathrm{mg}$ of $\mathrm{NO}_{3}{ }^{-}$promoted a decrease in SBP $(-6.2 \mathrm{~mm} \mathrm{Hg}), \mathrm{DBP}(-5.2 \mathrm{~mm} \mathrm{Hg})$, and heart rate $(-7 \mathrm{bpm})$ in a pilot study conducted with healthy individuals. However, $\mathrm{NO}_{3}{ }^{-}$supplementation had to be adjusted to treat hypertensive individuals, since similar doses in compromised vascular individuals do not alter hemodynamic parameters. The non-susceptibility of 27 treated hypertensive patients was clearly demonstrated by the intake of $7.0 \mathrm{mmol}(434 \mathrm{mg})$ of $\mathrm{NO}_{3}{ }^{-}$in $140 \mathrm{~mL}$ of beetroot juice for 7 days, resulting in increased $\mathrm{NO}$ synthesis, assessed by plasmatic, urinary and salivary $\mathrm{NO}_{3}{ }^{-}$and $\mathrm{NO}_{2}{ }^{-}$, but with no differences in home and $24 \mathrm{~h}$ ambulatory, SBP and DBP [47]. These results indicate that, in order to ameliorate primary hemodynamic parameters, high doses of dietary $\mathrm{NO}_{3}{ }^{-}$combined with a long-term intervention can be applied to treat individuals presenting impaired endothelial function. Furthermore, in an unprecedented clinical trial, patients displaying at least three risk factors for the development of CVD, including hypertension, were chronically supplemented for three weeks with an enriched $\mathrm{NO}_{3}{ }^{-}$beetroot-cereal bar providing $589 \mathrm{mg}$ of $\mathrm{NO}_{3}{ }^{-}$in $60 \mathrm{~g}$ of the intervention product, resulting in $14.0 \mathrm{~mm} \mathrm{Hg}$ and $6.5 \mathrm{~mm} \mathrm{Hg}$ decreases in SBP and DBP, respectively, in response to $\sim 15$-fold or $\sim 7$-fold increased plasma $\mathrm{NO}_{3}{ }^{-}$and $\mathrm{NO}_{2}{ }^{-}$ concentrations, respectively. Endothelial function in the treated volunteers was improved and arterial stiffness was reduced by $14 \%[45,51]$.

\section{Plasma $\mathrm{NO}_{3}{ }^{-} / \mathrm{NO}_{2}{ }^{-}$Increments on Cardiovascular Health and Impaired Cardiovascular Functions}

It is known that plasma $\mathrm{NO}_{3}{ }^{-}$and $\mathrm{NO}_{2}{ }^{-}$concentrations are dependent on ingested $\mathrm{NO}_{3}{ }^{-}$[52], but the minimum increase in $\mathrm{NO}_{3}{ }^{-} / \mathrm{NO}_{2}{ }^{-}$plasma levels necessary to promote hemodynamic responses may differ between healthy individuals and those with compromised cardiovascular function. In a clinical trial where healthy men received dietary supplementation, 3.5-fold and 1.6-fold increases of plasma $\mathrm{NO}_{3}{ }^{-}$and $\mathrm{NO}_{2}{ }^{-}$, respectively, 
resulted in significant DBP reductions and increases in endothelium-independent vasodilatation. This small but effective plasma increase was generated after the acute intake of beetroot bread $\left(\mathrm{NO}_{3}{ }^{-} 1.1 \mathrm{mmol}\right)$ [39]. On the other hand, Haun et al. [49] reported plasma $\mathrm{NO}_{\mathrm{x}}\left(\sim 3\right.$-fold) and $\mathrm{NO}_{2}{ }^{-}$(less than 1.5-fold) increases, albeit without any changes in hemodynamic parameters such as heart rate, DBP, SBP, FMD, radial artery pulse waves (PWA), central mean arterial pressure (CMAP) and central pulse pressure (CPP), after the acute intake of red spinach extract $\left(\mathrm{NO}_{3}{ }^{-} 1.45 \mathrm{mmol}\right)$ by 15 healthy subjects. Although the dose used by Haun et al. [49] was slightly higher than by Hobbs et al. [39] trial, plasma $\mathrm{NO}_{3}{ }^{-}$ (>3.5-fold) and $\mathrm{NO}_{2}{ }^{-}$(>1.6-fold) increases should be a determinant factor in choosing the dose required to benefit healthy populations.

In individuals with impaired cardiovascular function, the administered $\mathrm{NO}_{3}{ }^{-}$dose should be able to meet two requirements: (i) promote an increase in systemic $\mathrm{NO}_{3}{ }^{-}$and $\mathrm{NO}_{2}{ }^{-}$higher than observed in healthy individuals; (ii) be administered in a chronic and uninterrupted manner.

Hypertensive pregnant women exhibited $\sim 10$ - and $~ 1.5$-fold increases in plasma $\mathrm{NO}_{3}{ }^{-}$ and $\mathrm{NO}_{2}{ }^{-}$, respectively, after 7 days of daily supplementation with $\mathrm{NO}_{3}{ }^{-}(6.45 \mathrm{mmol}$ in beetroot juice). No significant differences were observed in plasma $\mathrm{NO}_{3}{ }^{-}$and $\mathrm{NO}_{2}{ }^{-}$levels measured $24 \mathrm{~h}$ after the initial dose, and even in the following 7-days of daily supplementation [53]. Similarly, a 1-week intake of beetroot juice $\left(\mathrm{NO}_{3}{ }^{-} \sim 6.45 \mathrm{mmol}\right)$ in 27 treated hypertensive individuals resulted in a three-fold increase in plasma $\mathrm{NO}_{3}{ }^{-}$and $\mathrm{NO}_{2}{ }^{-}$, with no differences in home and 24-h ambulatory blood pressures [54]. Finally, 24 overweight older subjects supplemented for 3 weeks with concentrated beet juice $(\sim 4.8-6.45 \mathrm{mmol})$ exhibited urinary $\mathrm{NO}_{3}{ }^{-}$values $\sim 3$-fold higher greater than the baseline and beneficial SBP effects after juice intake. However, both urinary $\mathrm{NO}_{3}{ }^{-}$and SBP returned to baseline levels $24 \mathrm{~h}$ after ingestion and in the first week following the end of supplementation [40]. These findings demonstrate that acute treatments able to promote systemic increases in $\mathrm{NO}_{3}{ }^{-}$and $\mathrm{NO}_{2}{ }^{-}$at levels similar to those observed in healthy individuals do not benefit individuals presenting cardiovascular risks.

On the other hand, clinical trials lasting more than 3 weeks or comprising higher $\mathrm{NO}_{3}{ }^{-}$doses than usually applied (6-7 mmol) resulted in better hemodynamic outcomes. Hypertensive subjects treated for 4 weeks with beetroot juice $\left(\mathrm{NO}_{3}{ }^{-} 6.4 \mathrm{mmol}\right)$ exhibited substantial increases in $\mathrm{NO}_{3}{ }^{-}$and $\mathrm{NO}_{2}{ }^{-}$plasma levels ( 5.5 and $\sim 2.7$, respectively). In addition, this intervention provided sustained BP lowering of $7.7 / 5.2 \mathrm{~mm} \mathrm{Hg} 24 \mathrm{~h}$ after the treatment, with clinical BP reduced by $7.7 / 2.4 \mathrm{~mm} \mathrm{Hg}$ and home BP, by $8.1 / 3.8 \mathrm{~mm} \mathrm{Hg}$ [41]. In this trial, SBP and DBP reduction peaks occurred only in the last week, highlighting the importance of a prolonged intervention.

In another trial, supplementation for 3 weeks with a high dose of dietary $\mathrm{NO}_{3}{ }^{-}$ concentrate in a $60 \mathrm{~g}$ beetroot cereal bar $(9.5 \mathrm{mmol})$ resulted in $\sim 15$ - and $\sim 7$-fold increases in plasma $\mathrm{NO}_{3}{ }^{-}$and $\mathrm{NO}_{2}{ }^{-}$, respectively. This was accompanied by a considerable reduction in BP $(-14.0 /-6.5 \mathrm{~mm} \mathrm{Hg})$ and improvement in central hemodynamic and endothelial function parameters such as arterial stiffness, augmentation and index pressures, aortic systolic and pulse pressures and cutaneous microvascular conductance [45].

Based on these reports, individuals presenting physiopathological conditions that affect the cardiovascular system require a dietary therapy that associates high $\mathrm{NO}_{3}{ }^{-}$doses capable of promoting systemic increases in $\mathrm{NO}_{3}{ }^{-}$and $\mathrm{NO}_{2}{ }^{-}$to levels higher than found in healthy individuals and in addition, is administered continuously (Figure 2). 

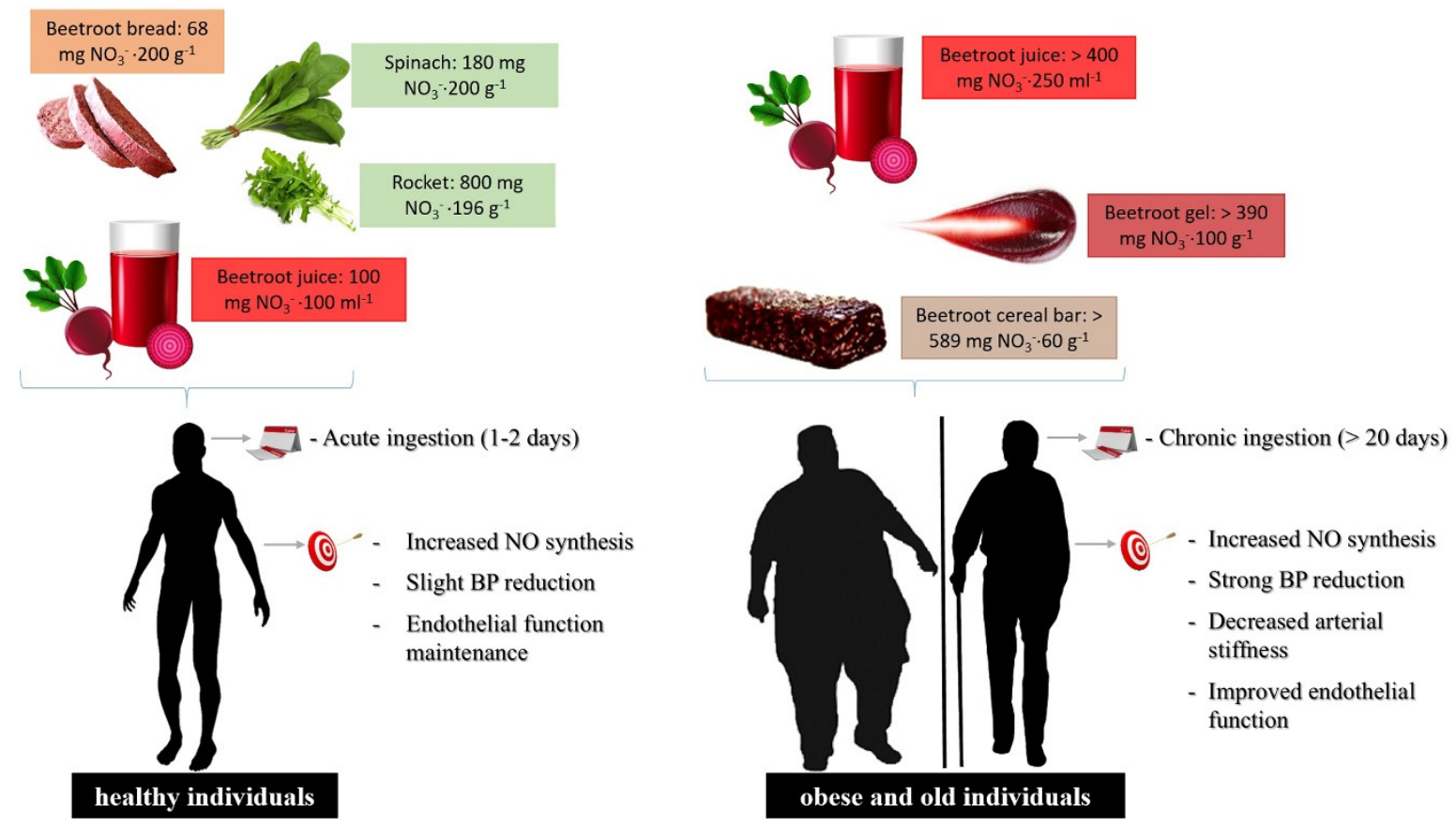

Figure 2. Food formulations and supplementation regimen of dietary $\mathrm{NO}_{3}{ }^{-}$in healthy or cardiovascular-compromised patients. For individuals presenting risk factors for the development of cardiovascular disease, the dietary $\mathrm{NO}_{3}{ }^{-}$dose should be higher to promote the systemic elevation of plasma $\mathrm{NO}_{3}{ }^{-}$and $\mathrm{NO}_{2}{ }^{-}$levels compared to healthy individuals, increasing $\mathrm{NO}$ generation by the $\mathrm{NO}_{3}{ }^{-} / \mathrm{NO}_{2}{ }^{-}$pathway, where increased levels must be administered through chronic and uninterrupted supplementation.

In short, the aforementioned studies discussed herein suggest that frequent daily dietary $\mathrm{NO}_{3}{ }^{-}$doses for long periods of time would be necessary to promote beneficial effects on blood pressure and endothelial function in populations presenting compromised vascular responsiveness. A systematic review and meta-analysis study of randomized controlled trials demonstrated that supplementation of inorganic $\mathrm{NO}_{3}{ }^{-}$from beetroot juice over 14 days provoked decreases in SBP $(-3.55 \mathrm{~mm} \mathrm{Hg}$; 95\% CI: $-4.55,-2.54 \mathrm{~mm} \mathrm{Hg})$ and DBP $(-1.32 \mathrm{~mm} \mathrm{Hg}$; $95 \% \mathrm{CI}:-1.97,-0.68 \mathrm{~mm} \mathrm{Hg})$. Furthermore, beneficial dietary $\mathrm{NO}_{3}{ }^{-}$effects on endothelial function were associated with dose, age, and body mass index (BMI), where chronic beetroot juice supplementation improved flow-mediated dilation (FMD) and endothelium functional effects according to the administered $\mathrm{NO}_{3}{ }^{-}$contents $(\beta=0.04, \mathrm{SE}=0.01, p<0.001)$, age $(\beta=-0.01, \mathrm{SE}=0.004, p=0.02)$ and $\mathrm{BMI}(\beta=-0.04$, $\mathrm{SE}=0.02, p=0.05$ ) [55]. A critical review of experimental data shows that chronic dietary $\mathrm{NO}_{3}{ }^{-}$ingestion is a positive vascular endothelium effector promoting vasodilatation and reducing blood pressure in compromised vascular responsiveness individuals.

However, only beetroot supplementation has been tested in acute and chronic assays in individuals with impaired cardiovascular function. Although the $\mathrm{NO}_{3}{ }^{-}$content of green leaves is able to fulfill the effective $\mathrm{NO}_{3}{ }^{-}$concentration in such patients, beetroot formulations may be the best non-drug strategy, since beetroot-derived formulations can concentrate the pharmacological $\mathrm{NO}_{3}{ }^{-}$dosage in a small serving portion of an attractive food product, favoring continuous intake and better adherence to this nutritional intervention. This may explain the well-documented and consistent cardioprotective effects of beet products in both healthy individuals and those presenting risk factors for the development of $\mathrm{CVD}$ when compared with other rich- $\mathrm{NO}_{3}{ }^{-}$vegetables, such as green leaves, assayed in clinical trials.

Furthermore, it is important to note that, in addition to $\mathrm{NO}_{3}$, vegetables are also a source of numerous phytochemicals able to increase eNOS activity in endothelial cells and contribute to NO synthesis $[56,57]$. Due to the great variety of polyphenols and other bioactive compounds in vegetables, it is difficult to point out individual or synergistic 
effects on $\mathrm{NO}$ generation. However, only $\mathrm{NO}_{3}{ }^{-}$has been directly associated to the cardioprotective effect, since it provides the physiological substrate for $\mathrm{NO}$ generation via the $\mathrm{NO}_{3}{ }^{-}-\mathrm{NO}_{2}{ }^{-} / \mathrm{NO}$ enterosalivary pathway [8]. The administration of the same food matrix, depleted in $\mathrm{NO}_{3}{ }^{-}$, used as a placebo in the clinical trials had no effect on $\mathrm{NO}$ synthesis and hemodynamic parameters, proving that $\mathrm{NO}_{3}{ }^{-}$is probably the active principle. The remaining phytochemicals after $\mathrm{NO}_{3}{ }^{-}$removal, including polyphenols, which are preserved in the placebo, may promote a discrete increase in NO production but it seems they are not effective in promoting hemodynamic improvements, similar to the effect observed when $\mathrm{NO}_{3}{ }^{-}$at concentrations under the pharmacological threshold is administered.

\section{Conclusions}

Vegetables are important health-promoting foods in a balanced diet, due to the presence of bioactive compounds, including dietary $\mathrm{NO}_{3}{ }^{-}$. Vegetables that belong to the green leaf group, such as rocket, green spinach, basil, radish, Swiss chard and bok choy, in addition to red beetroot, are considered the richest sources of dietary $\mathrm{NO}_{3}{ }^{-}$. Increasing dietary $\mathrm{NO}_{3}{ }^{-}$ingestion results in beneficial effects in many physiological and clinical settings. Several clinical interventions with different $\mathrm{NO}_{3}{ }^{-}$-rich vegetables have been reported as affecting metabolic and cardiovascular functions by increasing $\mathrm{NO}$ concentrations and improving endothelial function by reducing BP and arterial stiffness. However, minimal or no hemodynamic and vascular beneficial effects in healthy individuals have been observed following acute $\mathrm{NO}_{3}{ }^{-}$ingestion. To obtain the maximum cardioprotective effects of $\mathrm{NO}_{3}{ }^{-}$ intake, patient health status, as well as $\mathrm{NO}_{3}{ }^{-}$dosage and supplementation regimen, must be considered.

The aforementioned studies suggest that frequent daily doses up to $6.0 \mathrm{mmol}$ of dietary $\mathrm{NO}_{3}{ }^{-}$for long periods of time ( $\geq 3$ weeks) are required to promote beneficial blood pressure and endothelial function effects, mainly in populations with compromised vascular responsiveness such as hypertensive, metabolic syndrome, obese and older individuals.

Only beetroot supplementation has been tested in acute and chronic assays in individuals with impaired cardiovascular function. Although the $\mathrm{NO}_{3}{ }^{-}$content of green leaves or other vegetables could fulfill the effective $\mathrm{NO}_{3}{ }^{-}$concentration in healthy individuals, patients with impaired vascular function require a higher dose able to provide systemic increases in $\mathrm{NO}_{3}{ }^{-}$and $\mathrm{NO}_{2}{ }^{-}$to levels higher than those achieved in healthy individuals. Beet formulations are easier, attractive, accessible and were the only vegetable shown to be effective in promoting increased systemic NO production at the magnitude necessary to achieve the expected pharmacological effects in individuals presenting cardiovascular disease risk factors.

Author Contributions: Conceptualization and study design, D.d.S.B., D.V.T.d.S. and V.M.F.P.; writing, the original draft preparation, D.d.S.B. and D.V.T.d.S.; writing, reviewing and editing V.M.F.P.; supervision V.M.F.P. All authors have read and agreed to the published version of the manuscript.

Funding: This research was funded by Coordenação de Aperfeiçoamento de Pessoal de Nível Superior (CAPES, Finance Code 001, grant No. 1627392) and Fundação Carlos Chagas Filho de Amparo à Pesquisa do Estado do Rio de Janeiro (FAPERJ grant No. E-26/202.815/2018, FAPERJ, PDR-10-grant No. E-26/202.254/2018 and grant No. E-26/202.345/2019).

Institutional Review Board Statement: Not applicable.

Informed Consent Statement: Not applicable.

Data Availability Statement: Not applicable.

Acknowledgments: The authors acknowledge financial support from Coordenação de Aperfeiçoamento de Pessoal de Nível Superior and Fundação Carlos Chagas Filho de Amparo à Pesquisa do Estado do Rio de Janeiro.

Conflicts of Interest: The authors declare no conflict of interest. 


\section{References}

1. Storch, A.S.; Mattos, J.D.; Alves, R.; Galdino, I.S.; Rocha, H.N.M. Methods of endothelial function assessment: Description and applications. Int. J. Cardiovasc. Sci. 2017, 30, 262-273. [CrossRef]

2. Lai, W.K.C.; Kan, M.Y. Homocysteine-induced endothelial dysfunction. Ann. Nutr. Metab. 2015, 67, 1-12. [CrossRef]

3. Krüger-Genge, A.; Blocki, A.; Franke, R.P.; Jung, F. Vascular endothelial cell biology: An update. Int. J. Mol. Sci. 2019, 20 , 4411. [CrossRef] [PubMed]

4. Carrillo, J.A.; Zafrilla, M.P.; Marhuenda, J. Cognitive Function and Consumption of Fruit and Vegetable Polyphenols in a Young Population: Is There a Relationship? Foods 2019, 8, 507. [CrossRef] [PubMed]

5. Ghosh, A.; Gao, L.; Thakur, A.; Siu, P.M.; Lai, C.W.K. Role of free fatty acids in endothelial dysfunction. J. Biomed. Sci. 2017, 24, 1-15. [CrossRef] [PubMed]

6. World Health Organization. Fruit and Vegetables for Health: Report of a Joint FAP/WHO Workshop; World Health Organization: Geneva, Switzerland, 2004; Available online: https:/ / apps.who.int/iris/handle/10665/43143 (accessed on 15 February 2021).

7. U.S. Department of Health and Human Services; U.S. Department of Agriculture. 2015-2020 Dietary Guidelines for Americans; U.S. Department of Health and Human Services/U.S. Department of Agriculture: Washington, DC, USA, 2015. Available online: https:/ / health.gov / our-work/food-and-nutrition/2015-2020-dietary-guidelines/ (accessed on 17 February 2021).

8. Lidder, S.; Webb, A. Vascular effects of dietary nitrate (as found in green leafy vegetables and beetroot) via the nitrate-nitrite-nitric oxide pathway. Br. J. Clin. Pharmacol. 2013, 75, 677-696. [CrossRef] [PubMed]

9. Baião, D.S.; da Silva, D.V.T.; Del Aguila, E.M.; Paschoalin, V.M.F. Nutritional, bioactive and physicochemical characteristics of different beetroot formulations. Food Addit. 2017, 20-44. [CrossRef]

10. Karwowska, M.; Kononiuk, A. Nitrates/nitrites in food-Risk for nitrosative stress and benefits. Antioxidants 2020, 9, 241. [CrossRef]

11. Hobbs, A.J.; Moncada, S. Antiplatelet properties of a novel, non-NO-based soluble guanylate cyclase activator. Vasc. Pharmacol. 2003, 40, 149-154. [CrossRef]

12. Stringham, J.M.; Stringham, N.T. Nitric Oxide and Lutein: Function, Performance, and Protection of Neural Tissue. Foods 2015, 4 , 678-689. [CrossRef]

13. Lundberg, J.O.; Gladwin, M.T.; Ahluwalia, A.; Benjamin, N.; Bryan, N.S.; Butler, A.; Cabrales, P.; Fago, A.; Feelisch, M.; Ford, P.C.; et al. Nitrate and nitrite in biology, nutrition and therapeutics. Nat. Chem. Biol. 2009, 5, 865-869. [CrossRef]

14. Bryan, N.S. Cardioprotective actions of nitrite therapy and dietary considerations. Front. Biosci. 2009, 14, 4793-4808. [CrossRef]

15. Donato, A.J.; Morgan, R.G.; Walker, A.E.; Lesniewski, L.A. Cellular and molecular biology of aging endothelial cells. J. Mol. Cell. Cardiol. 2015, 89, 122-135. [CrossRef] [PubMed]

16. Virdis, A. Endothelial dysfunction in obesity: Role of inflammation. High Blood Press. Cardiovasc. Prev. 2016, 23, 83-85. [CrossRef] [PubMed]

17. Daiber, A.; Steven, S.; Weber, A.; Shuvaev, V.V.; Muzykantov, V.R.; Laher, I.; Li, H.; Lamas, S.; Münzel, T. Targeting vascular (endothelial) dysfunction. Br. J. Pharmacol. 2017, 174, 1591-1619. [CrossRef] [PubMed]

18. Hasler, C.M. The changing face of functional foods. J. Am. Coll. Nutr. 2000, 19, 499S-506S. [CrossRef] [PubMed]

19. Mohamed, S. Functional foods against metabolic syndrome (obesity, diabetes, hypertension and dyslipidemia) and cardiovascular disease. Trends Food Sci. Technol. 2014, 35, 114-128. [CrossRef]

20. Vulić, J.J.; Ćebović, T.N.; Čanadanović-Brunet, J.M.; Ćetković, G.S.; Čanadanović, V.M.; Djilas, S.M.; Šaponjac, V.T.T. In vivo and in vitro antioxidant effects of beetroot pomace extracts. J. Funct. Foods 2014, 6, 168-175. [CrossRef]

21. Thakur, N.; Raigond, P.; Singh, Y.; Mishra, T.; Singh, B.; Lal, M.K.; Dutt, S. Recent updates on bioaccessibility of phytonutrients. Trends Food Sci. Technol. 2020, 97, 366-380. [CrossRef]

22. Borgi, L.; Muraki, I.; Satija, A.; Willett, W.C.; Rimm, E.B.; Forman, J.P. Fruit and vegetable consumption and the incidence of hypertension in three prospective cohort studies. Hypertension 2016, 67, 288-293. [CrossRef]

23. Aune, D.; Giovannucci, E.; Boffetta, P.; Fadnes, L.T.; Keum, N.; Norat, T.; Greenwood, D.C.; Riboli, E.; Vatten, L.J.; Tonstad, S. Fruit and vegetable intake and the risk of cardiovascular disease, total cancer and all-cause mortality: A systematic review and dose-response meta-analysis of prospective studies. Int. J. Epidemiol. 2017, 46, 1029-1056. [CrossRef] [PubMed]

24. Van Breda, S.G.J.; de Kok, T.M.C.M. Smart combinations of bioactive compounds in fruits and vegetables may guide new strategies for personalized prevention of chronic diseases. Mol. Nutr. Food Res. 2018, 62, 1700597. [CrossRef] [PubMed]

25. Sawicki, T.; Martinez-Villaluenga, C.; Frias, J.; Wiczkowski, W.; Peñas, E.; Baczek, N.; Zieliński, H. The effect of processing and in vitro digestion on the betalain profile and ACE inhibition activity of red beetroot products. J. Funct. Foods 2019, 55, 229-237. [CrossRef]

26. Mirvish, S.S. Role of N-nitroso compounds (NOC) and N-nitrosation in etiology of gastric, esophageal, nasopharyngeal and bladder cancer and contribution to cancer of known exposures to NOC. Cancer Lett. 1995, 93, 17-48. [CrossRef]

27. Katan, M.B. Nitrate in foods: Harmful or healthy? Am. J. Clin. Nutr. 2009, 90, 11-12. [CrossRef] [PubMed]

28. Blekkenhorst, L.C.; Bondonno, N.P.; Liu, A.H.; Ward, N.C.; Prince, R.L.; Lewis, J.R.; Devine, A.; Croft, K.D.; Hodgson, J.M.; Bondonno, C.P. Nitrate, the oral microbiome, and cardiovascular health: A systematic literature review of human and animal studies. Am. J. Clin. Nutr. 2018, 107, 504-522. [CrossRef] 
29. Alidadi, M.; Jamialahmadi, T.; Cicero, A.F.G.; Bianconi, V.; Pirro, M.; Banach, M.; Sahebkar, A. The potential role of plant-derived natural products in improving arterial stiffness: A review of dietary intervention studies. Trends Food Sci. Technol. 2020, 99, 426-440. [CrossRef]

30. Baião, D.S.; da Silva, D.V.T.; Paschoalin, V.M.F. Beetroot, a remarkable vegetable: Its nitrate and phytochemical contents can be adjusted in novel formulations to benefit health and support cardiovascular disease therapies. Antioxidants 2020, 9, 960. [CrossRef]

31. Anjana, S.U.; Muhammad, I.; Abrol, Y.P. Are nitrate concentrations in leafy vegetables within safe limits? Curr. Sci. 2007, 92, 355-360. Available online: http:/ / www.jstor.org/stable/24096732 (accessed on 5 March 2021).

32. Blekkenhorst, L.C.; Sim, M.; Bondonno, C.P.; Bondonno, N.P.; Ward, N.C.; Prince, R.L.; Devine, A.; Lewis, J.R.; Hodgson, J.M. Cardiovascular health benefits of specific vegetable types: A narrative review. Nutrients 2018, 10, 595. [CrossRef]

33. Hord, N.G.; Tang, Y.; Bryan, N.S. Food sources of nitrates and nitrites: The physiologic context for potential health benefits. Am. J. Clin. Nutr. 2009, 90, 1-10. [CrossRef] [PubMed]

34. Santamaria, P.; Elia, A.; Serio, F.; Todaro, E. A survey of nitrate and oxalate content in fresh vegetables. J. Sci. Food Agric. 1999, 79, 1882-1888. [CrossRef]

35. European Food Safety Authority (EFSA). Opinion of the scientific panel on contaminants in the food chain on a request from the European Commission to perform a scientific risk assessment on nitrate in vegetables. EFSA J. 2008, 689, 1-79. [CrossRef]

36. Tamme, T.; Reinik, M.; Roasto, M.; Meremäe, K.; Kiss, A. Nitrate in leafy vegetables, culinary herbs, and cucumber grown under cover in Estonia: Content and intake. Food Addit. Contam. Part B 2010, 3, 108-113. [CrossRef] [PubMed]

37. van Velzen, A.G.; Sips, A.J.; Schothorst, R.C.; Lambers, A.C.; Meulenbelt, J. The oral bioavailability of nitrate from nitrate-rich vegetables in humans. Toxicol. Lett. 2008, 181, 177-181. [CrossRef] [PubMed]

38. Hobbs, D.A.; Kaffa, N.; George, T.W.; Methven, L.; Lovegrove, J.A. Blood pressure-lowering effects of beetroot juice and novel beetroot-enriched bread products in normotensive male subjects. Br. J. Nutr. 2012, 108, 2066-2074. [CrossRef] [PubMed]

39. Hobbs, D.A.; Goulding, M.G.; Nguyen, A.; Malaver, T.; Walker, C.F.; George, T.W.; Methven, L.; Lovegrove, J.A. Acute ingestion of beetroot bread increases Endothelium-Independent vasodilation and lowers diastolic blood pressure in healthy men: A randomized controlled trial. J. Nutr. 2013, 143, 1399-1405. [CrossRef] [PubMed]

40. Jajja, A.; Sutyarjoko, A.; Lara, J.; Rennie, K.; Brandt, K.; Qadir, O.; Siervo, M. Beetroot supplementation lowers daily systolic blood pressure in older, overweight subjects. Nutr. Res. 2014, 34, 868-875. [CrossRef] [PubMed]

41. Kapil, V.; Khambata, R.S.; Robertson, A.; Caulfield, M.J.; Ahluwalia, A. Dietary nitrate provides sustained blood pressure lowering in hypertensive patients: A randomized, phase 2, double-blind, placebo-controlled study. Hypertension 2015, 65, 320-327. [CrossRef]

42. Baião, D.S.; Conte-Junior, C.A.; Paschoalin, V.M.F.; Alvares, T.S. Beetroot juice increase nitric oxide metabolites in both men and women regardless of body mass. Int. J. Food Sci. Nutr. 2016, 67, 40-46. [CrossRef]

43. Bezerra, A.D.L.; Costa, E.C.; Pacheco, D.A.; Souza, D.C.; Farias-Junior, L.F.; Ritti-Dia, R.M.; Grigolo, G.B.; Júnior, P.I.H.B.; Krause, M.; Fayh, A.P.T. Effect of acute dietary nitrate supplementation on the post-exercise ambulatory blood pressure in obese males: A randomized, controlled, crossover trial. J. Sports Sci. Med. 2019, 18, 118-127.

44. Silva, D.V.T.; Silva, F.O.; Perrone, D.; Pierucci, A.P.T.R.; Conte-Junior, C.A.; Alvares, T.S.; Del Aguila, E.M.; Paschoalin, V.M.F. Physicochemical, nutritional, and sensory analyses of a nitrate-enriched beetroot gel and its effects on plasmatic nitric oxide and blood pressure. Food Nutr. Res. 2016, 60, 29909. [CrossRef]

45. Baião, D.S.; d'El-Rei, J.; Alves, G.; Neves, M.F.; Perrone, D.; Del Aguila, E.D.; Paschoalin, V.M.F. Chronic effects of nitrate supplementation with a newly designed beetroot formulation on biochemical and hemodynamic parameters of individuals presenting risk factors for cardiovascular diseases: A pilot study. J. Funct. Foods 2019, 58, 85-94. [CrossRef]

46. Liu, A.H.; Bondonno, C.P.; Croft, K.D.; Puddey, I.B.; Woodman, R.J.; Rich, L.; Ward, N.C.; Vita, J.A.; Hodgson, J.M. Effects of a nitrate-rich meal on arterial stiffness and blood pressure in healthy volunteers. Nitric Oxide 2013, 35, 123-130. [CrossRef]

47. Bondonno, C.P.; Yang, X.; Croft, K.D.; Considine, M.J.; Ward, N.C.; Rich, L.; Puddey, I.B.; Swinny, E.; Mubarak, A.; Hodgson, J.M. Flavonoid-rich apples and nitrate-rich spinach augment nitric oxide status and improve endothelial function in healthy men and women: A randomized controlled trial. Free Radic. Biol. Med. 2012, 52, 95-102. [CrossRef] [PubMed]

48. Jonvik, K.L.; Nyakayiru, J.; Pinckaers, P.J.; Senden, J.M.; van Loon, L.J.; Verdijk, L.B. Nitrate-rich vegetables increase plasma nitrate and nitrite concentrations and lower blood pressure in healthy adults. J. Nutr. 2016, 146, 986-993. [CrossRef]

49. Haun, C.T.; Kephart, W.C.; Holland, A.M.; Mobley, C.B.; McCloskey, A.E.; Shake, J.J.; Pascoe, D.D.; Roberts, M.D.; Martin, J.S. Differential vascular reactivity responses acutely following ingestion of a nitrate rich red spinach extract. Eur. J. Appl. Physiol. 2016, 116, 2267-2279. [CrossRef]

50. Ashor, A.W.; Jajja, A.; Sutyarjoko, A.; Brandt, K.; Qadir, O.; Lara, J.; Siervo, M. Effects of beetroot juice supplementation on microvascular blood flow in older overweight and obese subjects: A pilot randomised controlled study. J. Hum. Hypertens. 2015, 29, 511-513. [CrossRef]

51. Baião, D.S.; Silva, F.O.; d'El-Rei, J.; Neves, M.F.; Perrone, D.; Del Aguila, E.M.; Paschoalin, V.M.F. A new functional beetroot formulation enhances adherence to nitrate supplementation and health outcomes in clinical practice. SDRP J. Food Sci. Technol. 2018, 3, 484-496. [CrossRef] 
52. Webb, A.J.; Patel, N.; Loukogeorgakis, S.; Okorie, M.; Aboud, Z.; Misra, S.; Rashid, R.; Miall, P.; Deanfield, J.; Benjamin, N.; et al. Acute blood pressure lowering, vasoprotective, and antiplatelet properties of dietary nitrate via bioconversion to nitrite. Hypertension 2008, 51, 784-790. [CrossRef]

53. Ormesher, L.; Myers, J.E.; Chmiel, C.; Wareing, M.; Greenwood, S.L.; Tropea, T.; Lundberg, J.O.; Weitzberg, E.; Nihlen, C.; Sibley, C.P.; et al. Effects of dietary nitrate supplementation, from beetroot juice, on blood pressure in hypertensive pregnant women: A randomized, double-blind, placebo-controlled feasibility trial. Nitric Oxide 2018, 80, 37-44. [CrossRef] [PubMed]

54. Bondonno, C.P.; Liu, A.H.; Croft, K.D.; Ward, N.C.; Shinde, S.; Moodley, Y.; Lundberg, J.O.; Puddey, I.B.; Woodman, R.J.; Hodgson, J.M. Absence of an effect of high nitrate intake from beetroot juice on blood pressure in treated hypertensive individuals: A randomized controlled trial. Am. J. Clin. Nutr. 2015, 102, 368-375. [CrossRef]

55. Lara, J.; Ashor, A.W.; Oggioni, C.; Ahluwalia, A.; Mathers, J.C.; Siervo, M. Effects of inorganic nitrate and beetroot supplementation on endothelial function: A systematic review and meta-analysis. Eur. J. Nutr. 2016, 55, 451-459. [CrossRef] [PubMed]

56. Ambriz-Pérez, D.L.; Leyva-López, N.; Gutierrez-Grijalva, E.P.; Heredia, J.B. Phenolic compounds: Natural alternative in inflammation treatment. A Review. Cogent. Food Agric. 2016, 2, 1-14. [CrossRef]

57. Appeldoorn, M.M.; Venema, D.P.; Peters, T.H.F.; Koenen, M.E.; Arts, I.C.W.; Vincken, J.P.; Gruppen, H.; Keijer, J.; Hollman, P.C.H. Some Phenolic Compounds Increase the Nitric Oxide Level in Endothelial Cells in Vitro. J. Agric. Food Chem. 2009, 57, 7693-7699. [CrossRef] 